\title{
Publisher Correction: A note on the Lena image
}

Correction to: Nature Nanotechnology https://doi.org/10.1038/s41565-018-0337-2, published online 6 December 2018.

In the version of this Editorial originally published, the sentence "Like the Lena image, all these alternatives are in the public domain" was incorrect; it should have said that the alternatives "are widely available". This has now been corrected in the online versions of the Editorial.

Published online: 10 June 2019

https://doi.org/10.1038/s41565-019-0490-2 